\title{
Detecção do Hipotiveoidismo Subclínico em Gestantes Com Diferentes Idades Gestacionais
}

\section{RESUMO}

\begin{abstract}
Objetivo: Detectar hipotireoidismo subclínico em gestantes. Material e Método: Foram estudadas 75 gestantes, voluntárias, residentes na cidade de Itabuna, Bahia. O protocolo constou de: critérios de inclusão: gestante com faixa etária $\leq 40$ anos, sem história prévia de doença tireoidiana ou auto-imunes e diabetes mellitus em qualquer idade gestacional; avaliação clínica com entrevista sob a forma de questionário; avaliação laboratorial com dosagem de $\mathrm{T}_{4}$ livre, TSH, anticorpos anti-TPO, perfil lipídico (colesterol total, HDL-colesterol e triglicérides); avaliação ultra-sonográfica da tireóide. Resultados: A idade média foi de 21,6 6 5, 1 anos (14-40); a idade gestacional média foi de 24,2 \pm 8,2 semanas (5-39); foi encontrado TSH elevado com $\mathrm{T}_{4}$ livre normal em 3 gestantes (4,0\%). A positividade de anticorpos anti-TPO foi de 8,0\%. Foram encontradas 5,4\% de alterações ultra-sonográficas tireoidianas. Conclusão: Encontramos uma prevalência de $4,0 \%$ de hipotireoidismo subclínico na amostra, e com base neste resultado os autores consideram de grande importância a inclusão da avaliação tireoidiana na rotina do exame pré-natal. Estudos ulteriores se fazem necessários para estabelecer em que momento a avaliação tireoidiana de mulheres grávidas deve ser iniciada e com que freqüência deve ser repetida durante o curso da gestação. (Arq Bras Endocrinol Metab 2005;49/6:923-929)
\end{abstract}

Descritores: Hipotireoidismo subclínico; Função tireoidiana; Gestante; Tireóide

\section{ABSTRACT}

Detection of Subclinical Hypothyroidism in Pregnant Women With Different Gestational Ages.

Aim: To detect subclinical hypothyroidism in pregnant women. Subjects and Methods: Seventy-five pregnant women who resided in the town of Itabuna, state of Bahia, were voluntarily studied. Inclusion criteria were age $\leq 40$ years, no history of previous thyroid disease, autoimmunopathy or diabetes mellitus, and any gestational age; a clinical evaluation (an interview obeying to a questionnaire); laboratory evaluation (free $\mathrm{T}_{4}, \mathrm{TSH}_{\text {, }}$ anti-TPO antibody, total and HDL cholesterol, triglyceride determinations); thyroid ultrasonography. Results: Average age was 21.6 \pm 5.1 (14-40 years); gestation age was $24.2 \pm 8.2$ (5-39 weeks); an elevated TSH with normal free $\mathrm{T}_{4}$ was found in 3 cases (4.0\%). Anti-TPO antibodies were positive in $8.0 \%$ on the pregnant women. In $5.4 \%$ of them, thyroid ultrasonographic changes were documented. Conclusion: Based on finding of a $4 \%$ prevalence of elevated TSH during pregnancy, the authors consider important the inclusion of thyroid function laboratory evaluation in the routine prenatal examination. Further studies appear necessary to establish at what gestational age thyroid function evaluation should be started in pregnant women and how frequently it should be repeated during the course of gestation. (Arq Bras Endocrinol Metab 2005;49/6:923-929)

Keywords: Subclinical hypothyroidism; Thyroid function; Pregnant woman; Thyroid artigo original

\author{
Luis J.O. Andrade \\ Thomaz Cruz \\ Carla Daltro \\ Caroline S. França \\ Avelina O.S. Nascimento
}

Curso de Pós-Graduação em Medicina e Saúde, Universidade Federal da Bahia, Salvador, BA.

Recebido em 18/10/04 Revisado em 29/03/05 e 10/06/05 Aceito em 16/09/05 
$\mathbf{O}$ HIPOTIREOIDISMO SUBCLÍNICO É UMA CONDIÇÃO em que se encontram elevações séricas do hormônio estimulante da tireóide (TSH) $(>5 \mu \mathrm{UI} / \mathrm{ml}$ ), na vigência de níveis séricos normais de hormônios tireoidianos (1). O aumento dos níveis de TSH estimula a glândula tireóidea a produzir mais hormônios tireoidianos e, nestes casos, enquanto houver tecido tireoidiano responsivo, ocorrerá normalização da produção hormonal tireoidiana, caracterizando assim o hipotireoidismo subclínico (2).

Durante o período gestacional é de extrema importância o diagnóstico precoce de qualquer doença, especialmente aquelas sem sintomatologia aparente, ou com sintomas que se confundam com os da gravidez e nos quais o diagnóstico é essencialmente laboratorial, como as chamadas doenças subclínicas ou minimamente sintomáticas. O obstetra deve ter especial atenção a doenças como o hipotireoidismo, tendo em vista as conseqüências para o concepto e para a própria gestante.

A gestação leva a modificações funcionais e estruturais na tireóide que simulam alterações patológicas; ela interfere na fisiologia tireoidiana através de várias vias. $\mathrm{O}$ eixo hipotálamo-hipófise-tireóide materno durante o período gestacional sofre uma série de adaptações, bem como o feto irá desenvolver também seu próprio eixo, e a placenta irá desempenhar um papel ativo no transporte e metabolismo do iodo e da tiroxina $\left(\mathrm{T}_{4}\right)$, de modo que, no curso da gestação há três vias integradas atuando na fisiologia tireoidiana. Inicialmente ocorre um aumento de duas a três vezes da globulina transportadora de $\mathrm{T}_{4}$ (TBG) decorrente de um aumento da sua produção hepática e também devido à diminuição da sua depuração (aumento do ácido siálico em sua molécula), conseqüente ao aumento dos estrógenos circulante. Assim, a dosagem do $\mathrm{T}_{4}$ total aumenta, contudo sua fração livre permanece normal. Em seguida, devido ao aumento da gonodotrofina coriônica, ocorre um aumento dos níveis séricos de triiodotironina $\mathrm{e}_{4} \mathrm{~T}$ suas frações livres no primeiro trimestre.

$\mathrm{O}$ TSH pode apresentar-se diminuído no primeiro trimestre, decorrente da ação "TSH-simile" da hCG, retornando aos níveis normais ou mesmo elevados ao final da gestação. A redução do TSH coincide com o aumento das hCG, especialmente entre a oitava e a décima quarta semanas de gestação $(6,7)$.

Outro dado a ser analisado, é a relação feto-placentária. Classicamente, considerava-se que o $\mathrm{T}_{3}$ e o $\mathrm{T}_{4}$ não atravessavam a barreira feto-placentária, por isso não se dava importância à possibilidade de a função tireoidiana da gestante influenciar o desen- volvimento fetal. Entretanto, evidências clínicas (8) e experimentais (9) demonstraram a importância da passagem transplacentária de hormônios tireoidianos no desenvolvimento fetal, principalmente no sistema nervoso central. Assim é que o $\mathrm{T}_{4}$ tem sido mensurado no líquido celômico humano desde a $4^{\mathrm{a}}$ semana de gestação (10), sendo detectado no sangue do cordão umbilical de recém-nascidos com agenesia ou disgenesia tireoidiana (11).

Atualmente, publicações têm chamado atenção ao papel da condição hormonal tireoidiana materna no prognóstico neuropsicológico fetal após o nascimento (12). Tem sido proposto rastreamento no sentido de detectar hipotireoidismo ou hipotireoidismo subclínico em gestantes (13), com o objetivo de reconhecer riscos e prevenir prejuízos.

$\mathrm{Na}$ gestação, o hipotireoidismo clínico tem uma prevalência de 0,3 a $0,7 \%$ (14); já estudos sobre o hipotireoidismo subclínico, relatados na literatura, apresentam uma prevalência bem maior em relação ao hipotireoidismo manifesto, com índice de prevalência em torno de 2,5\% (15).

Em mulheres não grávidas, há controvérsia ainda sobre a necessidade de tratamento do hipotireoidismo subclínico ou qual a melhor ocasião para tratá-lo, porém em gestante é obrigatório seu tratamento, tendo em vista as evidências com relação ao déficit no desenvolvimento neuropsicomotor do concepto (12) e a possibilidade de a gestante desenvolver hipotireoidismo clínico, especialmente na presença de anticorpo anti-peroxidase (anti-TPO) (16). Portanto, o tratamento do hipotireoidismo subclínico é essencial e seguro tanto para a gestante quanto para o feto (17).

Tendo em vista estas particularidades na função tireoidiana das gestantes, e pelo fato de a avaliação tireoidiana não fazer parte de rotina do pré-natal, o objetivo deste trabalho foi realizar um estudo descritivo da freqüência de hipotireoidismo subclínico em uma amostra de gestantes.

\section{MATERIAL E MÉTODOS}

Trata-se de um estudo descritivo de corte transversal. O protocolo do estudo foi aprovado pelo comitê de ética em pesquisa da Santa Casa de Itabuna, Bahia.

A população de referência constitui-se de gestantes de ambulatórios de pré-natal da rede pública $(86,7 \%)$ e de clínicas privadas $(13,3 \%)$. Constou de 75 gestantes, adotando-se como critérios de inclusão idade menor que 40 anos e em qualquer idade gestacional. Adotou-se como critérios de exclusão: ges- 
tantes com doença auto-imune diagnosticada, com diabetes mellitus ou diabetes gestacional e com história pregressa de doença e/ou cirurgia tireoidiana. Como critério diagnóstico de hipotireoidismo subclínico foi adotado nível sérico de TSH maior que $5 \mu \mathrm{UI} / \mathrm{ml}$ com nível de $\mathrm{T}_{4}$ livre normal.

A avaliação da função tireoidiana foi realizada via a determinação do TSH e do $\mathrm{T}_{4}$ livre, que foram medidos por ensaios imunoenzimáticos (Baxter Diagnostics Inc., Deerfield, EUA - sistema automatizado ACS-180 ${ }^{\circledR}$, com sensibilidade funcional média de $0,05 \mu \mathrm{UI} / \mathrm{mL}$ e $0,4 \mathrm{ng} / \mathrm{dL}$ respectivamente. Os coeficientes de variação intra e inter-ensaio são de $5,3 \%$ e $5,0 \%$ para o $\mathrm{TSH}$ e de $6,0 \%$ e $7,0 \%$ para o $\mathrm{T}_{4}$ livre. Os valores referenciais normais para o TSH foram de 0,27 a $4,2 \mu \mathrm{UI} / \mathrm{mL}$ e para o $\mathrm{T}_{4}$ livre foram de 0,93 a $1,7 \mathrm{ng} / \mathrm{dL}$.

Os anticorpos anti-TPO foram quantificados pelo método de imunoensaio de eletroquimioluminescência (DYNOtest anti-TPO, BRAHMS Diagnostica $^{\circledR}$, Berlin, Alemanha) com limite de sensibilidade > $0 \mathrm{UI} / \mathrm{ml}$. O coeficiente de variação foi de $7,2 \%$ e $12,2 \%$. O valor de referência normal, até $34 \mathrm{UI} / \mathrm{mL}$.

As determinações do colesterol total e triglicérides foram realizadas pelo método enzimático colorimétrico, por meio de sistema automatizado SELECTRA II Merck ${ }^{\circledR}$, tendo, como valores de referência, menor que $200 \mathrm{mg} / \mathrm{dL}$ para o colesterol e menor que $150 \mathrm{mg} / \mathrm{dL}$ para os triglicérides. O coeficiente de variação foi de $1,28 \%$ e $1,58 \%$ para o colesterol e de $0,78 \%$ e $1,08 \%$ para os triglicérides.

As medidas do HDL-colesterol foram realizadas pelo método colorimétrico direto, por meio de sistema automatizado SELECTRA II Merck ${ }^{\circledR}$, tendo como valores de referência para mulheres 45 a $65 \mathrm{mg} / \mathrm{dL}$, e coeficiente de variação de $0,79 \%$ e $0,88 \%$.

A avaliação ultra-sonográfica da glândula tireóidea foi realizada pelo mesmo observador, por meio de equipamento Diasonic Gateway 2D ${ }^{\circledR}$ (USA), com transdutor linear de $10 \mathrm{MHz}$ de freqüência, através de contato direto.

\section{ANÁLISE ESTATÍSTICA}

As variáveis analisadas foram idade, paridade, idade gestacional no dia do exame, dados clínicos (sensação de frio, raciocínio lento, memória fraca, sonolência, pele seca, queda de cabelo, prisão de ventre, bradicardia, sensação de fraqueza, bócio e rouquidão, pressão arterial e pulso), dados laboratoriais $\left(\mathrm{T}_{4}\right.$ livre, $\mathrm{TSH}$, anticorpo anti-TPO, colesterol total, HDL colesterol e triglicérides) e ultra-sonografia da tireóide.
Após a revisão dos questionários preenchidos, os dados foram codificados e digitados, utilizando-se o programa Statistical Package for the Social Sciences (SPSS Chicago - IL, versão 9.0).

Para considerar uma variável com tendo distribuição normal, foram utilizados os seguintes parâmetros: média, mediana, moda, desvio-padrão, simetria e achatamento da curva, histograma, Q-Q plots e teste de Kolmogoroff-Smirnov para normalidade.

Os resultados das variáveis contínuas foram apresentados sob a forma de média \pm desvio-padrão ou mediana e amplitude interquartil.

Para comparação das variáveis entre dois grupos foi utilizado o teste de Mann-Whitney. Para comparação de proporções foi utilizado o teste exato de Fisher.

Todos os testes foram aplicados utilizando-se intervalo de confiança de $95 \%$. Todos os testes foram bi-caudais e só aplicados após verificação das premissas para sua utilização.

\section{RESULTADOS}

As gestantes apresentaram idade média de 21,5 $\pm 5,1$ anos, mediana de 21 anos, variando de 14 a 40 anos de idade.

Quanto à paridade, 38 gestantes eram primigestas $(50,7 \%), 25$ eram secundigestas $(33,3 \%)$ e 12 gestantes eram multíparas $(16,0 \%)$, sendo $7(9,3 \%)$ na terceira gestação, $2(2,7 \%)$ na quarta gestação, $2(2,7 \%)$ na quinta gestação e $1(1,3 \%)$ na sexta gestação.

A idade gestacional média na amostra foi de $24,2 \pm 8,2$ semanas, sendo que $50 \%$ das gestantes tinham idade gestacional maior ou igual a 25 semanas na época da entrevista, variando de 5 a 39 semanas de gestação (gráfico 1).

No questionário de avaliação das gestantes foram incluídos sinais e sintomas comuns ao hipotireoidismo (manifesto ou subclínico) e à gestação. Os principais sinais e sintomas referidos pelas gestantes, quando da avaliação clínica realizada, foram, por ordem decrescente: 1. sonolência - 64,0\%; 2 . queda de cabelo $-52,0 \% ; 3$. sensação de fraqueza $44,0 \% ; 4$. memória fraca $-36,0 \% ; 5$. prisão de ventre $30,7 \%$; 6. raciocínio lento $-29,3 \%$; 7. pele seca $28,0 \% ; 8$. sensação de frio $-24,0 \% ; 9$. bradicardia $4 \%$; 10 . bócio $-4 \%$; 11 . rouquidão $-4 \%$.

Verificou-se para os níveis séricos de $\mathrm{T}_{4}$ livre uma média de $1,05 \pm 0,23 \mathrm{ng} / \mathrm{dL}$, com mediana de $0,99 \mathrm{ng} / \mathrm{dL}$, variando de 0,76 a $2,4 \mathrm{ng} / \mathrm{dL}$. Das 75 gestantes estudadas, $3(4 \%)$ apresentaram $\mathrm{T}_{4}$ livre elevado (maior que $1,7 \mathrm{ng} / \mathrm{dL}$ - valor referência máximo 


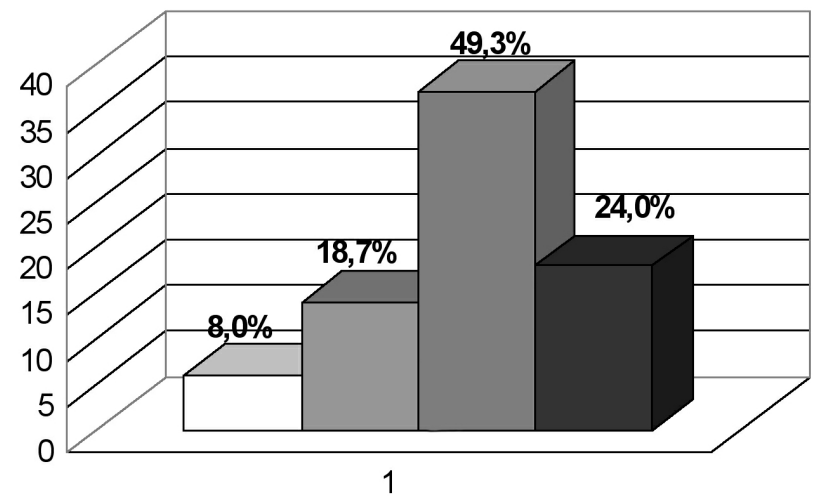

Gráfico 1. Idade gestacional na população estudada.

Tabela 1. Niveis de $\mathrm{T}_{4}$ livre e TSH na amostra avaliada.

\begin{tabular}{lcccc}
\hline & N & Média \pm DP & Variações & Valor Referência \\
\hline $\mathrm{T}_{4}$ livre $(\mathrm{ng} / \mathrm{dL})$ & 75 & $1,05 \pm 0,23$ & $0,76-2,4$ & $0,93-1,7$ \\
$\mathrm{TSH}(\mu \mathrm{Ul} / \mathrm{mL})$ & 75 & $2,35 \pm 1,03$ & $0,28-6,25$ & $0,27-4,2$ \\
\hline
\end{tabular}

para o método utilizado), enquanto que 2 gestantes apresentaram $\mathrm{T}_{4}$ livre diminuído (menor que 0,93 ng/dL - valor referência mínimo para o método utilizado). Das 5 gestantes que apresentaram alterações dos níveis de $\mathrm{T}_{4}$ livre, os níveis de $\mathrm{TSH}$ encontravamse na faixa de normalidade.

Para o TSH obteve-se uma média de 2,35 \pm $1,03 \mu \mathrm{UI} / \mathrm{mL}$, com mediana de $2,10 \mu \mathrm{UI} / \mathrm{mL}$, variando de 0,28 a 6,25. Das 75 gestantes estudadas, 3 (4\%) apresentaram TSH sérico maior que $4,2 \mu \mathrm{UI} / \mathrm{mL}$ (valor referência máximo para o método utilizado), todas com níveis de $\mathrm{T}_{4}$ livre normais e apenas uma com anti-TPO positivo, e apresentavam idade gestacional à época do exame de 18,21 e 28 semanas.

Com base nos valores de referência até 34 $\mathrm{UI} / \mathrm{mL}$ para o método utilizado na avaliação de anticorpos anti-TPO, verificou-se uma positividade em seis gestantes $(8 \%)$, enquanto que em 69 gestantes $(92 \%)$ os resultados ou foram negativos $(82,7 \%)$ ou estavam abaixo do nível superior de referência $(9,3 \%)$. Observou-se que os níveis variaram de negativo a 110 $\mathrm{UI} / \mathrm{mL}$, média de 7,3 $\pm 23,6 \mathrm{UI} / \mathrm{mL}$, mediana negativa. Das seis gestantes que apresentaram positividade do anti-TPO, apenas uma apresentou nível sérico de TSH acima dos valores de referência.

$\mathrm{Na}$ amostra analisada observou-se um colesterol total com média de $197,0 \pm 35,3 \mathrm{mg} / \mathrm{dL}$, com mediana de $193,0 \mathrm{mg} / \mathrm{dL}$, variando de 138 a $336 \mathrm{mg} / \mathrm{dL}$. Cinqüenta e quatro gestantes (72\%) apresentaram colesterol total abaixo de $200 \mathrm{mg} / \mathrm{dL}$ (valor máximo de referência no método utilizado), enquanto que $28 \%$ (21 gestantes) apresentaram colesterol total acima do valor de referência máximo. Das gestantes que apresentaram colesterol total elevado, 5 apresentaram HDL-colesterol abaixo dos valores referenciais e 14 apresentaram triglicérides elevados. Observou-se, ainda, no grupo de gestantes com colesterol total acima dos valores referenciais, duas gestantes com anticorpos anti-TPO positivo e três com TSH acima dos valores de referência.

Os níveis da fração HDL-colesterol apresentaram uma média de $48,1 \pm 5,4 \mathrm{mg} / \mathrm{dL}$, com mediana de $48,0 \mathrm{mg} / \mathrm{dL}$, com variação de 37,0 a $80,0 \mathrm{mg} / \mathrm{dL}$. Da amostra estudada, $85,4 \%$ das gestantes apresentaram HDL-colesterol entre $45-65 \mathrm{mg} / \mathrm{dL}$ (valores referenciais ), $13,3 \%$ abaixo de $45 \mathrm{mg} / \mathrm{dL}$ e $1,3 \%$ acima de $65 \mathrm{mg} / \mathrm{dL}$.

Os níveis de triglicérides apresentaram uma média de $152,4 \pm 39,6 \mathrm{mg} / \mathrm{dL}$, com mediana de $145,0 \mathrm{mg} / \mathrm{dL}$, variando de 80,0 a $349,0 \mathrm{mg} / \mathrm{dL}$. Da amostra estudada, $60 \%$ apresentaram triglicérides abaixo do valor referencial máximo de $150 \mathrm{mg} / \mathrm{dL}$. Das trinta gestantes que apresentaram triglicérides acima do valor de referência, quatro apresentaram anticorpos anti-TPO positivo, enquanto que duas gestantes apresentaram TSH acima dos valores referenciais.

Os resultados da avaliação ultra-sonográfica da amostra de gestantes estudadas evidenciaram uma fre- 
qüência de 71 exames normais, correspondendo a 94,6\%, tendo $2,7 \%$ das gestantes apresentado aumento do volume tireoidiano e em 2,7\% detectou-se cisto colóide.

\section{DISCUSSÃO}

Na literatura médica brasileira não existem trabalhos sobre o estudo da prevalência de hipotireoidismo subclínico em gestantes. A primeira referência concernente ao tema foi feita na década de 60 do século passado (18). Nos últimos anos, entretanto, após o estudo de Haddow e cols. (12), tem crescido a importância do diagnóstico do agravo em questão, tendo em vista suas conseqüências relatadas no estudo, referentes ao desenvolvimento neuropsicomotor dos filhos das gestantes portadoras do hipotireoidismo subclínico que não foram tratadas no curso da gravidez.

De modo geral, as anormalidades da função tireoidiana durante a gestação podem afetar até 10\% de todas as gestantes. A alta prevalência de disfunções tireoidianas, associadas às repercussões obstétricas nas gestantes, bem como o potencial papel da disfunção da tireóide materna influenciando o desenvolvimento fetal, constituem-se em forte argumento para a avaliação da função tireoidiana durante a gestação (14).

$\mathrm{Na}$ grande maioria das vezes, o quadro clínico do hipotireoidismo subclínico é pouco consistente, e na gestante apresenta como complicador o fato de sua sintomatologia, na sua maioria, ser semelhante ao da gestação normal, portanto o seu diagnóstico laboratorial e a determinação de sua prevalência são de fundamental relevância, tendo em vista a prevenção de complicações maternas e para o concepto.

A amostra estudada foi constituída por gestantes de quatro centros de saúde da cidade de Itabuna, Bahia, em sua maioria de baixa renda, como também de cinco clínicas privadas, estas de classe média.

Os níveis séricos de TSH na maioria das gestantes encontram-se na faixa de normalidade, porém pequenas variações podem ocorrer, apresentando uma tendência para a redução dos seus níveis durante o primeiro trimestre devido ao aumento da gonodotrofina coriônica $(19,20)$.

Trabalhos em diferentes países também mostraram que os níveis séricos de TSH durante a gravidez são dependentes da fonte de iodo disponível $(21,22)$. No Brasil, nos últimos 15 anos, tem-se adicionado teores superiores a $10 \mathrm{mg}$ de iodo $/ \mathrm{kg}$ de sal, o que tem levado à profilaxia da carência de iodo em todo país, praticamente em toda população, em especial nas gestantes que estariam recebendo doses adequadas de iodo (23).
Klein e cols., avaliando a função tireoidiana de 2.000 gestantes com idade gestacional entre 15 e 18 semanas, detectou 49 gestantes com níveis séricos de TSH maior que $6,0 \mu \mathrm{UI} / \mathrm{mL}$, sendo que em 43 os níveis séricos de $\mathrm{T}_{4}$ livre estavam dentro dos limites de normalidade (15). Em trabalho de publicação recente, foram avaliados o nível sérico de TSH de 17.298 gestantes com idade gestacional em torno de 20 semanas, sendo detectado $2,3 \%$ de hipotireoidismo subclínico, observando que estas gestantes apresentaram 3 vezes mais complicações em relação às normais, em função principalmente de descolamento de placenta e parto prematuro (24). Nossos dados mostraram que os níveis séricos de TSH das gestantes avaliadas variaram de 0,28 a $6,25 \mu \mathrm{UI} / \mathrm{mL}$, tendo $96 \%$ da amostra apresentado níveis dentro dos valores de referência, e $4 \%$ da amostra apresentado o TSH acima de $4,2 \mu \mathrm{UI} / \mathrm{mL}$ (valor de referência máximo para o método utilizado).

As concentrações dos hormônios tireoidianos livres refletem o real estado metabólico tireoidiano da gestante. Usualmente os níveis de $\mathrm{T}_{4}$ livre estão dentro dos limites de normalidade na maioria das gestantes, porém com inclinação para o limite superior de normalidade ou levemente acima do normal no inicio da gestação (25). Estudos em gestantes normais demonstraram que o $\mathrm{T}_{4}$ livre reduz para os valores referenciais mais baixos no $2^{\circ}$ e $3^{\circ}$ trimestres da gestação, em áreas com ou sem deficiência de iodo. Essa redução do $\mathrm{T}_{4}$ livre associa-se com a redução do $\mathrm{T}_{3}$ livre e um aumento do $\mathrm{T}_{3}$ reverso (26).

Nossos resultados mostraram que os níveis séricos de $\mathrm{T}_{4}$ livre nas gestantes estudadas variaram de 0,76 a $2,4 \mathrm{ng} / \mathrm{dL}$, com média de $1,05 \pm 0,23 \mathrm{ng} / \mathrm{dL}$. Das 75 gestantes avaliadas, 3 (4\%) apresentaram níveis elevados de $\mathrm{T}_{4}$ livre, com níveis de TSH normais e anti-TPO negativo.

No curso da gestação ocorre uma gradual diminuição dos títulos de anticorpos anti-TPO, com elevação no puerpério. Estudos demonstram que a prevalência de anticorpos anti-tireoidianos nas gestantes variam de 1,9 a $18,7 \%$ (27-29). A presença de elevados títulos de anticorpos anti-TPO no primeiro trimestre da gestação aumentam a possibilidade de tireoidite pós-parto (TPP), e mulheres com anti-TPO positivo apresentam risco relativo de 20 a 80 para desenvolver a TPP $(16,30)$. Entretanto, um estudo indica que uma entre quatro mulheres com TPP confirmada apresenta negatividade de anticorpos antiTPO durante a gestação (31).

Nossos achados são concordantes com os da literatura. Em nosso estudo, as gestantes apresentaram níveis detectáveis de anticorpos anti-TPO em 17,3\%, 
porém se considerarmos os valores referenciais do método utilizado na avaliação (até $34 \mathrm{UI} / \mathrm{mL}$ ), 8\% das gestantes apresentaram anticorpos anti-TPO acima dos valores de referência.

Em nosso estudo, os níveis séricos de colesterol total variaram de 138 a $336 \mathrm{mg} / \mathrm{dL}$, com média de $197,0 \pm 35,4 \mathrm{mg} / \mathrm{dL}$. Com relação aos níveis séricos da fração HDL-colesterol, nosso estudo apresentou uma média de $48,2 \pm 5,5 \mathrm{mg} / \mathrm{dL}$, com níveis séricos variando de 37 a $80 \mathrm{mg} / \mathrm{dL}$.

Apesar de a gestação ser um período em que aumenta a demanda de iodo, não existem evidências do aumento do volume tireoidiano durante a gravidez, desde que a ingesta de iodo seja adequada. Porém, existem evidências de que o volume da glândula tireóidea aumenta durante a gravidez, nas áreas em que a disponibilidade de iodo é moderada ou baixa $(25,32)$. O aumento do volume tireoidiano nesta situação pode ser inibido parcial ou complemente com a suplementação de $100 \mu \mathrm{g} /$ dia de iodo (33).

A doença nodular tireoidiana é uma condição comum na população adulta, sendo predominante nas mulheres. A presença de nódulo tireoidiano clinicamente detectável está estimada em 4 a 7\%, porém, com o uso rotineiro da ultra-sonografia, dependendo da sensibilidade do equipamento utilizado, há relatos de 10 a 60\% de detecção incidental de nódulos (34). Estima-se em $10 \%$ a prevalência de nódulos tireoidianos clinicamente detectáveis em gestantes, sendo a maioria deles benignos e não funcionantes (12). Essa prevalência aumenta significativamente com a utilização de exames ecográficos quando se considera a ocorrência de gestação prévia, levando a sugerir que a gravidez desempenha um papel importante no desenvolvimento de nódulo tireoidiano, principalmente em áreas com deficiência de iodo.

Em nosso estudo, utilizou-se a ultra-sonografia para avaliação morfológica da glândula tireóidea das gestantes, a qual evidenciou 94,6\% de normalidade, enquanto que $2,7 \%$ da amostra analisada apresentaram aumento de volume tireoidiano e $2,7 \%$ das gestantes apresentaram cisto colóide.

Quanto às variáveis categóricas, não houve diferença estatisticamente significante entre os grupos com TSH elevado e com TSH normal, significando que as manifestações clínicas nas gestantes não devem ser os únicos parâmetros no diagnóstico do hipotireoidismo subclínico.

Considerando os achados descritos, concluímos que, na amostra estudada, a prevalência de hipotireoidismo subclínico foi de $4 \%$, não houve alterações ultrasonográficas significantes, a prevalência de anticorpos
anti-TPO foi de $8 \%$, não houve diferença significante nos sinais e sintomas entre os grupos de gestantes (normal e com hipotireoidismo subclínico) e conseqüentemente as manifestações clínicas não devem ser usadas como parâmetros únicos no diagnóstico do hipotireoidismo subclínico. Os autores reconhecem que repetidas medidas do TSH teriam sido mais representativas de queda progressiva da função tireoidiana durante a gravidez, e que uma só determinação pode ser considerada um fator limitante para o presente estudo. No entanto, convém ressaltar que entre as pacientes com aumento do TSH já se encontram algumas com aumento do LDL-colesterol e triglicérides e redução do HDL colesterol, o que se constitui em fator de risco cardiovascular, se persistente.

Considerando, ainda, a importância do diagnóstico do hipotireoidismo subclínico e suas repercussões, e apoiando-se em diretrizes internacionais, algumas medidas que objetivam a prevenção e conseqüentemente a redução de complicações resultantes do hipotireoidismo subclínico em gestantes deveriam ser adotadas, tais como: determinação precoce do TSH sérico durante a gestação, repetida se aumentado e tratada, em todas as gestantes com bócio, elevados títulos de anticorpos anti-tireoidianos, história familiar de doença tireoidiana, qualquer história de outra doença endócrina auto-imune, ou sintomas sugestivos de hipotireoidismo (35).

\section{REFERÊNCIAS}

1. Huber G, Staub JJ, Meier C, Mitrache C, Guglielmetti M, Huber $P$, et al. Prospective study of the spontaneous course of subclinical hypothyroidism: prognostic value of TSH, thyroid reserve and thyroid antibodies. J Clin Endocrinol Metab 2002;87:3221-6.

2. Larsen PR, Davies TF, Hay ID. The thyroid gland. In: Wilson JD, Foster DW, Kronemberg HM, Larsen PR, editors. Williams textbook of endocrinology, 9th ed. Philadelphia: WB Saunders, 1998. p.389-516.

3. Burrow GN, Fisher DA, Larsen PR. Maternal and fetal thyroid function. N Engl J Med 1994;331:1072-8.

4. Burrow GN. Thyroid diseases in pregnancy. In: Burrow GN, Oppenheimer JH, Volpé R, editors. Thyroid function \& disease, Philadelphia: WB Saunders, 1989. p.292-322.

5. Glinoer D, Lemone M, Bordoux $P$, De Nayer $P$, Delange $\mathrm{F}$, Kinthaert $\mathrm{J}$, et al. Partial reversibility during late pos partum of thyroid abnormalities associated with pregnancy. J Clin Endocrinol Metab 1992;74:453-7.

6. Becks GP, Burrow GN. Thyroid disease in pregnancy. Med Clin N Am 1991;75:121-4.

7. Rabelo LM, Araújo L, Rabelo MM. Disfunções tiroidianas na gravidez. Arq Bras Endocrinol Metab 1995;39:184-7. 
8. Pop VJ, Brouwers EP, Vader HL, Vulsma T, van Baar AL, de Vijlder JJ. Maternal hypothyroxinaemia during early pregnancy and subsequent child development: a 3 year follow-up study. Clin Endocrinol (Oxf) 2003:59(3):282-8.

9. Morreale de Escobar $G$, Obregón MJ, Ruiz de Oña C, Calvo R, Escobar del Rey F. Outer ring iodothyronine deiodinases and thyroid hormone economy, responses to iodine deficiency in the rat fetus and neonate. Endocrinology 1991;129:2663-73.

10. Contempré B, Jauniaux E, Calvo R, Jurkovic D, Campbell $S$, Morreale de Escobar $G$. Detection of thyroid hormones in human embryonic cavities during the first trimester of pregnancy. J Clin Endocrinol Metab 1993;77:1719-22.

11. Vulsma T, Gons MH, de Vijlder JJ. Maternal-fetal transfer of thyroxine in congenital hypothyroidism due to a total organification defect or thyroid agenesis. N Engl J Med 1989:321:13-6.

12. Haddow JE, Palomaki GE, Allan WC, Williams JR, Knight GJ, O'Heir CE, et al. Maternal thyroid deficiency during pregnancy and subsequent neuropsychological development of the child. N Engl J Med 1999;341:549-55.

13. Cooper DS. Subclinical hypothyroidism. N Engl J Med 2001;345:260-5.

14. Glinoer D. The systematic screening and management of hypothyroidism and hyperthyroidism during pregnancy. Trends Endocrinol Metab 1998;10:403-11.

15. Klein RZ, Haddow JE, Faix JD, Brown RS, Hermos RJ, Pulkkinen $A$, et al. Prevalence of thyroid deficiency in pregnant women. Clin Endocrinol (Oxf) 1991;35:41-6.

16. Lazarus $\mathrm{JH}$, Ludgate $\mathrm{M}$, Parkes $\mathrm{AB}$. Autoimmune thyroiditis: repercussions during and after pregnancy. In: Pinchera A, Mann K, Hostalek $U$, eds. The thyroid and age. Stuttgart: Schattauer, 1998. p.15-27.

17. Abolavich M, Gutierrez S, Alcaraz G, Maccallini G, Garcia $A$, Levalle $O$. Overt and subclinical hypothyroidism complicating pregnancy. Thyroid 2002; 12:63-8.

18. Choufoer JC, van Rhijn M, Querido A. Endemic goiter in Western New Guineas. II. Clinical picture, incidence and pathogenesis of endemic cretinism. J Clin Endocrinol Metab 1965;25:385-402.

19. Burrow GN. Doenças da Tireóide. In: Burrow GN, Ferris $H$, editors. Complicaçōes clínicas durante a gravidez, $4^{a}$ ed. São Paulo: Roca, 1996. p.155-86.

20. Emerson $\mathrm{CH}$. Thyroid disease during and after pregnancy. In: Braverman LE, Utiger RD. Werner and Ingbar's The thyroid, $7^{\text {th }}$ ed. Philadelphia: Lippincott-Raven, 1996. p.1021-31.

21. Silva JE, Silva S. Interrelationships among serum thyroxine, triiodothyronine, reverse triiodothyronine, and thyroid-stimulating hormone in iodine-deficient pregnant women and their offspring: effects of iodine supplementation. J Clin Endocrinol Metab 1981;52:671-7.

22. Thilly $\mathrm{CH}$, Delange $\mathrm{F}$, Lagasse $\mathrm{R}$. Fetal hypothyroidism and maternal thyroid status in severe endemic goiter. J Clin Endocrinol Metab 1978:47:356-60.
23. Knobel M, Medeiros-Neto $G$. Moléstias associadas à carência de iodo. Arq Bras Endocrinol Metab 2004:48:53-61.

24. Casey BM, Dashe JS, Wells CE, McIntire DD, Byrd W, Leveno KJ, et al. Subclinical hypothyroidism and pregnancy outcomes. Obstet Gynecol 2005; 105:235-6.

25. Berghout $A$, Wiersinga AB. Thyroid size and thyroid function during pregnancy: an analysis. Eur J Endocrinol 1998; 138:536-42.

26. Berghout A, Endert E. Thyroid function thyroid size in women living in an iodine-replete area. Clinical Endocrinology 1994:41:375-9.

27. Barros AFP, Vaisman M. Tireoidite pós-parto: prevalência e evolucão clínica. Arq Bras Endocrinol Metab $1998 ; 42: 451-5$

28. Ward LS. Predizendo riscos para tireoidite pós-parto através da dosagem de anticorpos anti-TPO: exemplo de valor da pesquisa clínica. Arq Bras Endocrinol Metab 1998:42:411-2.

29. Netto LS, Coeli CM, Micmacher E, Mamede SC, Nazar LO, Correa EK, et al. Estudo longitudinal do eixo hipófisetireóide durante a gravidez. Arq Bras Endocrinol Metab 2004;48:493-8.

30. Amino N, Tada H, Hidata Y. Postpartum auto-imune thyroid syndrome: a model of aggravation of auto-imune disease. Thyroid 1999:9:705-13.

31. Kuijpens JL, Pop VJ, Vader HL, Drexhage HA, Wiersinga WM. Prediction of postpartum thyroid dysfunction: can it be improved? Eur J Endocrinol 1998; 139:36-43.

32. Glinoer D. What happens to the normal thyroid during pregnancy? Thyroid 1999:9:631-5.

33. Pedersen KM, Laurberg $P$, Iversen $E$. Amelioration of some pregnancy associated variation in thyroid function by iodine supplementation. J Clin Endocrinol Metab 1994;77:1078-83

34. Tan GH, Gharib H. Thyroid incidentalomas: management approaches to non-palpable nodules discovered incidentally on thyroid imaging. Ann Intern Med 1997; 126:226-31.

35. Cruz TRP. Disfunção tireoidiana pós-parto: etiologias diversas, diagnóstico diferencial precoce, diferença no tratamento, experiência pessoal (tese de livre docência). Faculdade de Medicina - Universidade Federal da Bahia. 2000. XIII, 54p.

\section{Endereço para correspondência:}

Luis Jesuíno de Oliveira Andrade

Rua São Marcelo 246

45600-700 Itabuna, BA

E-mail: ljoa@nuxnet.com.br 